Open J. Math. Sci., Vol. 2(2018),No.1, pp. 29 - 38

Website: https://pisrt.org/psr-press/journals/oms/

ISSN: 2523-0212 (Online) 2616-4906 (Print)

http://dx.doi.org/10.30538/oms2018.0015

\title{
COMBINATION OF HOMOTOPY PERTURBATION METHOD (HPM) AND DOUBLE SUMUDU TRANSFORM TO SOLVE FRACTIONAL KDV EQUATIONS
}

\author{
HAMOOD UR REHMAN ${ }^{1}$, MUHAMMAD SHOAIB SALEEM, AYESHA AHMAD
}

\begin{abstract}
In this work, we developed homotopy perturbation double Sumudu transform method (HPDSTM) which is obtained by combining homotopy perturbation method, double Sumudu transform and He's polynomials. The method is applied to find the solution of linear fractional one and two dimensional dispersive $\mathrm{KdV}$ and nonlinear fractional $\mathrm{KdV}$ equations to illustrate the reliability of the method. It is observed that the solutions obtained by the method converge rapidly to the exact solutions. This method is very powerful, and professional techniques for solving different kinds of linear and nonlinear fractional order differential equations.

Mathematics Subject Classification: 34K37, 26A33, 35R11.

Key words and phrases: Homotopy perturbation method; double Sumudu transform: He's polynomial; Caputo fractional derivative; fractional KdV equations.
\end{abstract}

\section{Introduction}

Fractional calculus is a field of applied mathematics that deals with derivatives and integrals of arbitrary orders. In recent years, considerable interest in fractional differential equation has been stimulated due to their numerous applications in the areas of physics and engineering. Many important phenomena in electromagnetics, acoustics, viscoelasticity, electrochemistry and material science are well described by fractional differential equation. Fractional differential equations are increasingly used to model problems in fluid mechanics, acoustics, biology, electromagnetism, diffusion, signal processing, and many other physical

Received 13 January 2018. Revised 09 March 2018.

1 Corresponding Author

(C) 2018 Hamood ur Rehman, Muhammad Shoaib Saleem, and Ayesha Ahmad. This is an open access article distributed under the Creative Commons Attribution License, which permits unrestricted use, distribution, and reproduction in any medium, provided the original work is properly cited. 
processes $[1,2]$. In the literature one can find a wide class of methods dealing with the problem of approximate solutions to problems described by nonlinear fractional differential equations, for instance, asymptotic methods and perturbation methods.

A great deal of effort has been expended over the last 10 years or so in attempting to and robust and stable numerical and analytical methods for solving fractional partial differential equations of physical interest. Several numerical methods have been introduced to solve differential equations, such as the homotopy perturbation method (HPM) $[3,4,5]$, the Modified homotopy perturbation method (MHPM) [6], the differential transform method (DTM) [7], the variational iteration method (VIM) [8, 9], the homotopy analysis method (HAM) $[10,11]$, the Sumudu decomposition method [12], the Adomian decomposition method [13,14] and reproducing kernel method [15, 16, 17]. Among these methods, the HPM is a universal approach which can be used to solve FODEs and FPDEs. On the other hand, various methods are combined with the homotopy perturbation method, such as the variational homotopy perturbation method [18]. Another such combination is the homotopy perturbation transformation method [19]. Another such approach is the use of Homo-Separation of variables for the solution of fractional partial differential equations [20, 21, 22]. There are numerous integral transforms such as the Laplace, Sumudu, Fourier, Mellino solve PDEs. Of these, the Laplace transformation and Sumudu transformation are the most widely used. The Sumudu transformation method is one of the most important transform method. Various methods are combined with the Sumudu transformation method such as the homotopy analysis Sumudu transform method (HASTM) [23]. Another example is the Sumudu decomposition method (SDM) [24].

Singh et al. [25] have made used of studying the solutions of linear and nonlinear partial differential equations by using the homotopy perturbation Sumudu transform method. The nonlinear terms can be easily handled by the use of He's polynomial. The use of He's polynomials in the nonlinear term was first introduced by Ghorbani [26, 27]. There are some important applications and work on fractional differential equation in literature $[28,29,30]$.

In this paper, we applied homotopy perturbation double Sumudu transform method to obtain the analytical exact and approximate solutions. The proposed algorithm provides the solution in a rapid convergent series which may lead to the solution in a closed form. The advantage of this method is its capability of combining two powerful methods for obtaining exact solutions for nonlinear equations. The HPDSTM is a combination of double Sumudu transform, HPM, and He's polynomials. 


\section{Basic definitions and auxiliary theorems related to Sumudu Transform and fractional calculus}

The Sumudu transform is an integral transform similar to the Laplace transform, introduced in the early 1990s by Watugala [31] to solve differential equations and control engineering problems. Note that following theorems and definitions will be used in the rest of the paper.

Definition 2.1. The Sumudu transform of a function $f(t)$ defined for all real number $t \geq 0$ is the function $F_{s}(u)$ defined by

$$
S_{t}[f(t),(u)]=F_{s}(u)=\int_{0}^{\infty} \frac{1}{u} e^{\frac{-t}{u}} f(t) d t
$$

Many of special properties of the Sumudu transform are mentioned and tabulated in $[32,33]$. Some special properties of the Sumudu transform are as follows:

(1) $S_{t}[1]=1$

(2) $S_{t}\left[\frac{t^{n}}{\Gamma(n+1)}\right]=u^{n}$

(3) $S_{t}[f(x) \mp g(x)]=S[f(x)] \mp S[g(x)]$

Definition 2.2. The double Sumudu transform of a function $f(x, t)$, defined for all real numbers $(x \geq 0, t \geq 0)$ is defined by

$$
F(u, v)=S_{x t}[f(x, t),(u, v)]=\frac{1}{u v} \iint_{0}^{\infty} e^{-\left(\frac{t}{v}+\frac{x}{u}\right)} f(x, t) d x d t
$$

In the same line of ideas, the double Sumudu transform of second partial derivative with respect to $x$ is of form [34].

$$
S_{x t}\left[\frac{\partial^{2} f(x, t)}{\partial x^{2}} ;(u, v)\right]=\frac{1}{u^{2}} F(u, v)-\frac{1}{u^{2}} F(0, v)-\frac{1}{u} \frac{\partial F(0, v)}{\partial x}
$$

Similarly, the double Sumudu transform of second partial derivative with respect to $t$ is of form [34]

$$
S_{x t}\left[\frac{\partial^{2} f(x, t)}{\partial t^{2}} ;(u, v)\right]=\frac{1}{v^{2}} F(u, v)-\frac{1}{v^{2}} F(u, 0)-\frac{1}{v} \frac{\partial F(u, 0)}{\partial t}
$$

Theorem 2.3. [32] Let $G(u)$ be the Sumudu transform of $f(t)$, such that

(1) $G(1 / s) / s$, is a meromorphic function, with singularities having $\operatorname{Re}(s) \leq$ $\gamma$ and

(2) There exists a circular region $G$ with radius $R$ and positive constants, $M$ and $k$ with $|G(1 / s) / s|<M R^{-k}$ : then the function $f(t)$ is given by

$$
S^{-1}[G(s)]=\frac{1}{2 \Pi \iota} \int_{\gamma-\iota \infty}^{\gamma+\iota \infty} \exp [s t] G\left(\frac{1}{s}\right) \frac{d s}{s}=\sum \text { residual }\left[e^{s t} \frac{G\left(\frac{1}{s}\right)}{s}\right]
$$


Definition 2.4. The Riemann-Liouville fractional integral operator of order $\alpha>0$, of a function $f(t) \in C_{\mu}, \mu \geq-1$ is defined as

$$
j^{\alpha} f(t)=\frac{1}{\Gamma(\alpha)} \int_{0}^{t}(t-\tau)^{\alpha-1} f(\tau) d \tau
$$

Definition 2.5. $[10,27]$ The fractional derivative of $f(t)$ in the caputo sense is defined as

$$
\begin{aligned}
D_{t}^{\alpha} f(t) & =j^{m-\alpha} D^{n} f(t) \\
& =\frac{1}{\Gamma(n-\alpha)} \int_{0}^{t}(t-\tau)^{m-\alpha-1} f^{m} \tau d(\tau)
\end{aligned}
$$

for $m-1<\alpha \leq m, m \in N$ and $t>0$ and $\Gamma(\alpha)$ is a Gamma function.

Lemma 2.6. If $m-1<\alpha \leq m, m \in N$ and $f \in C_{\mu}^{m}$, and $\mu \geq-1$, then

$$
j^{\alpha} D_{0}^{\alpha} f(x)=f(x)-\sum_{k=0}^{m-1} f^{k}\left(0^{+}\right) \frac{x^{k}}{k !}, x>0
$$

Definition 2.7. Assume that $f(x)$ is a function of $n$ variables $x_{i}, i=1, \cdots, n$ also of class $\mathrm{C}$ on $D \in R_{n}$

$$
a \partial_{x}^{\alpha} f=\frac{1}{\Gamma(m-\alpha)} \int_{a}^{x_{i}}\left(x_{i}-t\right)^{m-\alpha-1} \partial_{x_{i}}^{m} f\left(x_{j}\right) d t
$$

where $\partial_{x_{i}}^{m}$ is the usual partial derivative of integer order $m$.

Theorem 2.8. Let $\frac{\partial^{i+j} f(x, t)}{\partial t^{j} \partial x^{i}}, i=0,1, \cdots, n, j=0,1, \cdots, m$ be of exponential order; i.e $\left|\frac{\partial^{i+j} f(x, t)}{\partial t^{j} \partial x^{i}}\right|<M e^{\frac{x}{\tau_{1}}+\frac{t}{\tau_{2}}}$ for some $M, \tau_{1}, \tau_{2}>0$, then the double Sumudu transform of the Caputo fractional derivative with respect to $x$ is defined as follows

$$
S_{x t}\left[\frac{\partial^{n} f(x, t)}{\partial x^{n}}\right]=u^{-n} S_{x t}[f(x, t)]-\sum_{i=1}^{n-1} u^{i-n} S_{x t}\left[\frac{\partial^{i} f(0, t)}{\partial x^{i}}\right]
$$

The double Sumudu transform of the Caputo fractional derivative with respect to $t$ is defined as follows

$$
S_{x t}\left[\frac{\partial^{m} f(x, t)}{\partial t^{m}}\right]=u^{-m} S_{x t}[f(x, t)]-\sum_{j=1}^{m-1} u^{j-m} S_{x t}\left[\frac{\partial^{j} f(x, 0)}{\partial t^{j}}\right]
$$

\section{Solution by HPDSTM}

We illustrate the basic idea of this method, by considering a general fractional nonlinear non- homogeneous partial differential equation with the initial condition of the form of general form

$$
D_{t}^{\alpha} U(x, t)=L(U(x, t))+N(U(x, t))+f(x, t), \alpha>0
$$


subject to initial condition

$$
D_{0}^{k} U(x, 0)=g_{k},(k=0, \cdots, n-1)
$$

$D_{0}^{n} U(x, 0)=0$, where, $D_{t}^{\alpha}=\frac{\partial^{\alpha}}{\partial t^{\alpha}}$ denotes without loss of generality the Caputo fraction derivative operator, $f$ is a known function, $N$ is the general nonlinear fractional differential operator, and $L$ represents a linear fractional differential operator. Applying the double Sumudu Transform on both sides of (12), we obtain

$$
S_{x t}\left[D_{t}^{\alpha} U(x, t)\right]=S_{x t}[L(U(x, t))]+S_{x t}[N(U(x, t))]+S_{x t}[f(x, t)]
$$

Using the property of the double Sumudu transform, we have

$S_{x t}[U(x, t)]=u^{\alpha} S_{x t}[L(U(x, t))]+u^{\alpha} S_{x t}[N(U(x, t))]+u^{\alpha} S_{x t}[f(x, t)]+g(x, t)$

Now applying the double Sumudu inverse on both sided of (15), we obtain

$$
U(x, t)=S_{x t}^{-1}\left[u^{\alpha} S_{x t}[L(U(x, t))]+u^{\alpha} S_{x t}[N(U(x, t))]\right]+G(x, t)
$$

where $G(x, t)$ represents the term arising from the known function $f(x, t)$ and the initial condition [31]. Now apply the HPM

$$
U(x, t)=\sum_{n=0}^{\infty} P^{n} U_{n}(x, t)
$$

The nonlinear term can be decomposed into

$$
N U(x, t)=\sum_{n=0}^{\infty} p^{n} H_{n}(U)(x, t)
$$

Using the He's polynomial [32] given as

$$
H_{n}\left(U_{0}, \cdots, U_{n}\right)=\frac{1}{n !} \frac{\partial^{n}}{\partial p^{n}}\left[N\left(\sum_{j=0}^{\infty} p^{j} U_{j}(x, t)\right)\right]
$$

Substituting (17) and (18) in (16)

$$
\begin{aligned}
\sum_{n=0}^{\infty} P^{n} U_{n}(x, t)= & G(x, t)+P\left[S _ { x t } ^ { - 1 } \left[u^{\alpha} S_{x t}\left[L\left(\sum_{n=0}^{\infty} P^{n} U_{n}(x, t)\right)\right]\right.\right. \\
& \left.\left.+u^{\alpha} S_{x t}\left[N\left(\sum_{n=0}^{\infty} P^{n} U_{n}(x, t)\right)\right]\right]\right]
\end{aligned}
$$

which is the coupling of the double Sumudu transform and the HPM using He's polynomials [34]. Comparing the coefficients of like powers of $P$, the following approximations are obtained:

$$
\begin{aligned}
& P^{0}: U_{0}(x, t)=G(x, t) \\
& P^{1}: U_{1}(x, t)=S_{x t}^{-1}\left[u^{\alpha} S_{x t}\left[L\left(U_{0}(x, t)\right)+H_{0}(U)\right]\right] \\
& P^{2}: U_{2}(x, t)=S_{x t}^{-1}\left[u^{\alpha} S_{x t}\left[L\left(U_{1}(x, t)\right)+H_{1}(U)\right]\right]
\end{aligned}
$$




$$
\begin{aligned}
P^{3}: U_{3}(x, t)= & S_{x t}^{-1}\left[u^{\alpha} S_{x t}\left[L\left(U_{2}(x, t)\right)+H_{2}(U)\right]\right] \\
& \vdots \\
P^{n}: U_{n}(x, t)= & S_{x t}^{-1}\left[u^{\alpha} S_{x t}\left[L\left(U_{2}(x, t)\right)+H_{n-1}(U)\right]\right]
\end{aligned}
$$

Finally, we approximate the analytical solution $U(x, t)$ by truncated series [34]

$$
U(x, t)=\lim _{N \rightarrow \infty} \sum_{n=0}^{N} U_{n}(x, t)
$$

The above series solutions generally converge very rapidly $[34,35,36]$.

\section{Applications}

In this section, we apply this method for solving solution of one and two dimensional linear fractional dispersive $\mathrm{KdV}$ and nonlinear fractional KdV equations to illustrate the reliability of the method.

Example 4.1. Consider the linear one dimensional fractional dispersive $\mathrm{KdV}$ equation [37]

$$
\begin{aligned}
u_{t}^{\alpha}(x, t) & =-2 u_{x}-u_{x x x}, t>0 \\
u(x, 0) & =\sin x
\end{aligned}
$$

Following carefully the steps involved in the HPDSTM, we arrive at the following series solutions

$$
\begin{aligned}
& u_{0}(x, t)=\sin x \\
& u_{1}(x, t)=-\frac{t^{\alpha}}{\Gamma(\alpha+1)} \cos x \\
& u_{2}(x, t)=-\frac{t^{2 \alpha}}{\Gamma(2 \alpha+1)} \sin x \\
& u_{3}(x, t)=\frac{t^{3 \alpha}}{\Gamma(3 \alpha+1)} \cos x
\end{aligned}
$$

we obtained solution in the following form

$$
u(x, t)=\sin x-\frac{t^{\alpha}}{\Gamma(\alpha+1)} \cos x-\frac{t^{2 \alpha}}{\Gamma(2 \alpha+1)} \sin x+\frac{t^{3 \alpha}}{\Gamma(3 \alpha+1)} \cos x+\ldots
$$

When $\alpha \rightarrow 1$, we recover the following series approximation

$$
\begin{aligned}
& u(x, t)=\sin x-t \cos x-\frac{t^{2}}{2 !} \sin x+\frac{t^{3}}{3 !} \cos x+\ldots \\
& u(x, t)=\sin x \cos t-\cos x \sin t=\sin (x-t)
\end{aligned}
$$

Which is the exact solution of this problem. 
Example 4.2. Consider the linear fractional two dimensional dispersive KdV equation [37]

$$
\begin{aligned}
u_{t}^{\alpha}(x, y, t) & =-u_{x x x}-u_{y y y}, t>0 \\
u(x, y, 0) & =\cos (x+y)
\end{aligned}
$$

Following carefully the steps involved in the HPDSTM, we arrive at the following series solutions

$$
\begin{aligned}
& u_{0}(x, y, t)=\cos (x+y) \\
& u_{1}(x, y, t)=-2 \frac{t^{\alpha}}{\Gamma(\alpha+1)} \sin (x+y) \\
& u_{2}(x, y, t)=-4 \frac{t^{2 \alpha}}{\Gamma(2 \alpha+1)} \cos (x+y) \\
& u_{3}(x, y, t)=8 \frac{t^{3 \alpha}}{\Gamma(3 \alpha+1)} \sin (x+y)
\end{aligned}
$$$$
\vdots
$$

We obtained solution in the following form

$$
\begin{aligned}
u(x, y, t)= & \cos (x+y)-2 \frac{t^{\alpha}}{\Gamma(\alpha+1)} \sin (x+y)-4 \frac{t^{2 \alpha}}{\Gamma(2 \alpha+1)} \cos (x+y) \\
& +8 \frac{t^{3 \alpha}}{\Gamma(3 \alpha+1)} \sin (x+y)+\ldots
\end{aligned}
$$

When $\alpha \rightarrow 1$ we recover the following series approximation

$$
\begin{aligned}
u(x, y, t) & =\cos (x+y)-2 t \sin (x+y)-4 \frac{t^{2}}{2 !} \cos (x+y)+8 \frac{t^{3}}{3 !} \sin (x+y)+\ldots \\
& =\sin (x+y+2 t)
\end{aligned}
$$

Which is the exact solution of this problem.

Example 4.3. We consider the following nonlinear time fractional KdV equation $[38]$

$$
\begin{aligned}
u_{t}^{\alpha}(x, t) & =\left(u^{2}\right)_{x}+\left[u(u)_{x x}\right]_{x}, t>0, x>0,0<\alpha \leq 1 \\
u(x, 0) & =\sinh ^{2}\left(\frac{x}{2}\right)
\end{aligned}
$$

Applying the double Sumudu transform on both sided, we obtained the following.

$$
S_{x t}[u(x, t)]=S_{x t}\left[\sinh ^{2}\left(\frac{x}{2}\right)\right]+u^{\alpha} S_{x t}\left[\left(u^{2}\right)_{x}+\left[u(u)_{x x}\right]_{x}\right]
$$

Applying the inverse double Sumudu transform, we obtain the following

$$
u(x, t)=\sinh ^{2}\left(\frac{x}{2}\right)+S_{x t}^{-1}\left[u^{\alpha}\left[S_{x t}\left[\left(u^{2}\right)_{x}+\left[u(u)_{x x}\right]_{x}\right]\right]\right]
$$


Now applying the homotopy perturbation tecnique on the above equation we obtain the following

$$
\begin{aligned}
\sum_{n=0}^{\infty} P^{n} u_{n}(x, t)= & \sinh ^{2}\left(\frac{x}{2}\right)+P\left[S _ { x t } ^ { - 1 } \left[u ^ { \alpha } \left[S _ { x t } \left[\sum_{n=0}^{\infty} P^{n} H_{n}(u)\right.\right.\right.\right. \\
& \left.\left.\left.\left.+\sum_{n=0}^{\infty} P^{n} H_{n}^{1}(u)\right]\right]\right]\right]
\end{aligned}
$$

where $H_{n}(u)$ and $H_{n}^{1}(u)$ are He's polynomials that represent the nonlinear terms.

By comparing the cofficients of like powers $p$, we have

$$
\begin{aligned}
& P^{0}: u_{0}(x, t)=\sinh ^{2}\left(\frac{x}{2}\right) \\
& P^{1}: u_{1}(x, t)=S_{x t}^{-1}\left[u^{\alpha}\left[S_{x t}\left[H_{0}(u)+H_{0}^{1}(u)\right]\right]\right]=-\frac{t^{\alpha}}{4 \Gamma(\alpha+1)} \sinh (x) \\
& P^{2}: u_{2}(x, t)=S_{x t}^{-1}\left[u^{\alpha}\left[S_{x t}\left[H_{1}(u)+H_{1}^{1}(u)\right]\right]\right]=+\frac{t^{2 \alpha}}{8 \Gamma(2 \alpha+1)} \cosh (x)
\end{aligned}
$$

Consequently the third term of the HPDST solution for example 4.3 is given by $u(x, t)=\sinh ^{2}\left(\frac{x}{2}\right)-\frac{t^{\alpha}}{4 \Gamma(\alpha+1)} \sinh (x)+\frac{t^{2 \alpha}}{8 \Gamma(2 \alpha+1)} \cosh (x)$.

\section{Conclusion}

The aim of this work was to make use of the properties of the double Sumudu transform to solve linear and nonlinear fractional KdV problems. The basic idea of the method combines double Sumudu transform and the HPM using He's polynomial. This combination builds a strong method called the homotopy perturbation double Sumudu transform (HPDSTD). The HPDSTD is an analytical method and runs using the initial conditions only. HPDSTM is a very powerful and efficient method to find approximate solutions as well as numerical solutions.

\section{Competing Interests}

The authors do not have any competing interests in the manuscript.

\section{REFERENCES}

1. Beyer, H., \& Kempfle, S. (1995). Definition of physically consistent damping laws with fractional derivatives. ZAMM-Journal of Applied Mathematics and Mechanics/Zeitschrift fr Angewandte Mathematik und Mechanik, 75(8), 623-635.

2. He, J. H. (1999). Some applications of nonlinear fractional differential equations and their approximations. Bull. Sci. Technol, 15(2), 86-90. 
3. He, J. H. (1998). Approximate analytical solution for seepage flow with fractional derivatives in porous media. Computer Methods in Applied Mechanics and Engineering, 167(12), 57-68.

4. He, J. H. (2005). Limit cycle and bifurcation of nonlinear problems. Chaos, Solitons 85 Fractals, 26(3), 827-833.

5. He, J. H. (1997). A new approach to nonlinear partial differential equations. Communications in Nonlinear Science and Numerical Simulation, 2(4), 230-235.

6. Hesameddini, E., \& Latifizadeh, H. (2009). An optimal choice of initial solutions in the homotopy perturbation method. International Journal of Nonlinear Sciences and Numerical Simulation, 10(11-12), 1389-1398.

7. Miller, K. S., \& Ross, B. (1993). An Introduction to the Fractional Calculus and Fractional Differential Equations. John Wiley and Sons, New York.

8. Bhalekar, S., \& Daftardar-Gejji, V. (2008). New iterative method: application to partial differential equations. Applied Mathematics and Computation, 203(2), 778-783.

9. Daftardar-Gejji, V., \& Bhalekar, S. (2010). Solving fractional boundary value problems with Dirichlet boundary conditions using a new iterative method. Computers \& Mathematics with Applications, 59(5), 1801-1809.

10. Arafa, A. A. M., Rida, S. Z., \& Mohamed, H. Homotopy analysis method for solving biological population model. Commum. Theor. Phys, 56, 797-800.

11. Hilfer, R. (2000). Applications of fractional calculus in physics. Applications of Fractional Calculus in Physics. Edited by HILFER R. Published by World Scientific Publishing Co. Pte. Ltd.,. ISBN no. 9789812817747.

12. Eltayeb, H., \& Kilicman, A. (2012). Application of Sumudu Decomposition Method to Solve Nonlinear System of Partial Differential Equations. Abstract and Applied Analysis, Volume 2012, Article ID 412948, 13 pages.

13. Adomian, G. (1994). Solving Frontier Problems of Physics: The Decomposition Method. Kluwer Academic Publishers, Boston and London.

14. Cheng, J. F., \& Chu, Y. M. (2011). Solution to the linear fractional differential equation using Adomian decomposition method. Mathematical Problems in Engineering, 2011. Article ID 587068, 14 pages.

15. Akram G., \& Rehman H. U. (2013). Solutions of a Class of Sixth Order Boundary Value Problems Using the Reproducing Kernel Space. Abstract and Applied Analysis, Volume 2013 (2013), Article ID 560590, 8 pages.

16. Akram, G., \& Rehman, H. U. (2013). Numerical solution of eighth order boundary value problems in reproducing Kernel space. Numerical Algorithms, 62(3), 527-540.

17. Akram, G., \& ur Rehman, H. (2013). A Numerical Solution to the Nonlinear Fifth Order Boundary Value Problems. International Journal of Applied Science and Engineering, $11(4), 415-422$.

18. Noor, M. A., \& Mohyud-Din, S. T. (2008). Variational homotopy perturbation method for solving higher dimensional initial boundary value problems. Mathematical Problems in Engineering, 2008. Article ID 696734, 11 pages.

19. Kumar, S., Yildirim, A., Khan, Y., \& Wei, L. (2012). A fractional model of the diffusion equation and its analytical solution using Laplace transform. Scientia Iranica, 19(4), 11171123.

20. Karbalaie, A., Montazeri, M. M., \& Muhammed, H. H. (2012). New approach to find the exact solution of fractional partial differential equation. WSEAS Transactions on Mathematics, 11(10), 908-917.

21. Karbalaie, A., Hamid Muhammed, H., \& Erlandsson, B. E. (2013). Using Homo-Separation of variables for solving systems of nonlinear fractional partial differential equations. International Journal of Mathematics and Mathematical Sciences, 2013. Article ID 421378, 8 pages. 
22. Karbalaie, A., Muhammed, H. H., Shabani, M., \& Montazeri, M. M. (2014). Exact Solution of Partial Differential Equation Using Homo-Separation of Variables. International Journal of Nonlinear Science, 17(1), 84-90.

23. Rathore, S., Kumar, D., Singh, J., \& Gupta, S. (2012). Homotopy analysis Sumudu transform method for nonlinear equations. International Journal of Industrial Mathematics, 4(4), 301-314.

24. Kumar, D., Singh, J., \& Rathore, S. (2012). Sumudu decomposition method for nonlinear equations. In International Mathematical Forum 7(11), 515-521.

25. Singh, J., \& Devendra, S. (2011). Homotopy perturbation Sumudu transform method for nonlinear equations. Adv. Theor. Appl. Mech, 4(4), 165-175.

26. Ghorbani, A., \& Saberi-Nadjafi, J. (2007). He's homotopy perturbation method for calculating adomian polynomials. International Journal of Nonlinear Sciences and Numerical Simulation, 8(2), 229-232.

27. Ghorbani, A. (2009). Beyond Adomian polynomials: he polynomials. Chaos, Solitons 86 Fractals, 39(3), 1486-1492.

28. Talib, I., Belgacem, F. M., Asif, N. A., \& Khalil H.(2017). On mixedderivative type high dimensional multi-term fractional partial differential equations approximate solutions. AIP Conference Proceedings, 1798(1), 20-24.

29. Nuruddeen, R. I. (2017). Multiple soliton solutions for the $(3+1)$ conformable space-time fractional modified Korteweg-de-Vries equations. Journal of Ocean Engineering and Science, 3(1) 10-18.

30. Nuruddeen, R. I., Muhammad, L., Nass, A. M., \& Sulaiman, T. A. (2018). A Review of the Integral Transforms-Based Decomposition Methods and their Applications in Solving Nonlinear PDEs.Palestine Journal of Mathematics, 1(7),262-280.

31. Watugala, G. K. (1993). Sumudu transform: a new integral transform to solve differential equations and control engineering problems. Integrated Education, 24(1), 35-43.

32. Belgacem, F. B. M., \& Karaballi, A. A. (2006). Sumudu transform fundamental properties investigations and applications. International Journal of Stochastic Analysis, 2006. 1-23.

33. Baleanu, D. (2012). Fractional calculus: models and numerical methods (Vol. 3). World Scientific.

34. Singh, J., Kumar, D., \& Kiliman, A. (2013). Homotopy perturbation method for fractional gas dynamics equation using Sumudu transform. Abstract and Applied Analysis 2013, Article ID 934060, 8 pages.

35. Wu, G. C. (2011). Adomian decomposition method for non-smooth initial value problems. Mathematical and Computer Modelling, 54(9-10), 2104-2108.

36. Tchuenche, J. M., \& Mbare, N. S. (2007). An application of the double Sumudu transform. Applied Mathematical Sciences, 1(1-4), 31-39.

37. Kanth, A. R., \& Aruna, K. (2015). Solution of fractional third-order dispersive partial differential equations. Egyptian Journal of Basic and Applied Sciences, 2(3), 190-199.

38. Momani, S., Odibat, Z., \& Hashim, I. (2008). Algorithms for nonlinear fractional partial differential equations: a selection of numerical methods. Topological Methods in Nonlinear Analysis, 31(2), 211-226.

\section{Hamood ur Rehman}

Department of Mathematics, University of Okara, Okara, Pakistan.

e-mail: hamood84@gmail.com

Muhammad Shoaib Saleem

Department of Mathematics, University of Okara, Okara, Pakistan.

e-mail: shaby455@yahoo.com

Ayesha Ahmad

Department of Mathematics, University of Okara, Okara, Pakistan. 
e-mail: ayeshahmad691@gmail.com 